\title{
In-Vitro Biofilm Formation and Antimicrobial Resistance of Escherichia coli in Diabetic and Nondiabetic Patients
}

\author{
Sunayana Raya ${ }^{D},{ }^{1}$ Ankit Belbase, ${ }^{1}$ Laxmi Dhakal, ${ }^{1}$ Krishna Govinda Prajapati, ${ }^{2}$ \\ Reena Baidya, ${ }^{2}$ and Nabin kishor Bimali ${ }^{1}$ \\ ${ }^{1}$ Department of Microbiology, Goldengate International College, Battisputali, Kathmandu, Nepal \\ ${ }^{2}$ Department of Pathology, BґB Hospital, Gwarko, Lalitpur, Nepal \\ Correspondence should be addressed to Sunayana Raya; sunayana.raya@gmail.com
}

Received 29 April 2019; Revised 23 July 2019; Accepted 05 August 2019; Published 19 September 2019

Academic Editor: Hugh D. Smyth

Copyright ( 2019 Sunayana Raya et al. This is an open access article distributed under the Creative Commons Attribution License, which permits unrestricted use, distribution, and reproduction in any medium, provided the original work is properly cited.

\begin{abstract}
Background. Diabetic patients are more susceptible to urinary tract infection compared to nondiabetic patients, Escherichia coli being the most common uropathogen causing UTI. Unreasonable and incorrect antibiotic prescription for UTI in these patients may induce the development of antibiotic-resistant urinary pathogens resulting in delayed recovery and longer hospitalization. In addition to these, biofilm forming capacity of the pathogen may worsen the problem. The main aim of this cross-sectional study (conducted from March to September 2015) is to detect the biofilm forming capacity of UTI causing micro-organisms and compare the antibiotic resistance pattern of Escherichia coli, the most common cause of UTI, which will help the physician in choosing the best antibiotic. Method. Total of 1,099 clean-catch mid stream urine (CCMSU) was processed by standard microbiological technique; 182 were from the diabetic group and 917 nondiabetic. Following identification, all isolates were subjected to antibiotic susceptibility testing using modified Kirby-Bauer disc diffusion method. In-vitro biofilm forming capacity of the isolates were detected by Microtitre plate method. The data were analyzed using SPSS software 16. Result. Urinary tract infection was found to be significantly higher in diabetic patients (42.9\%) compared to nondiabetic patients (17.4\%) with Escherichia coli as the most common uropathogen in both diabetic and nondiabetic groups. Similarly, UTI was more common in elderly population (29.5\%). Imipenem, nitrofurantoin and amikacin were found to be the most effective drug for uropathogenic E. coli in both diabetic and nondiabetic patients, whereas amoxicillin, ciprofloxacin, and cotrimoxazole were least effective. Of the total bacterial isolates, $43.3 \%$ showed positive results for in-vitro biofilm production by the Microtitre plate method. A significantly higher resistance rate was observed among biofilm producing E. coli for quinolones, cotrimoxazole, and third generation cephalosporin ceftriaxone. Most of the biofilm producers (79.5\%) were found to be MDR ( $p$-value 0.015$)$. Conclusion. Elderly populations with diabetes are at a higher risk of UTI. Higher biofilm production and resistance to in-use antimicrobial agents in this study render its inefficacy for empirical treatment and point out the importance of biofilm screening to ensure the effective management of infection.
\end{abstract}

\section{Introduction}

Diabetes is a chronic, metabolic disease characterized by increased levels of blood glucose, which on overtime leads to serious damage to heart, kidney blood vessels, nerves, and eyes. The number of people with diabetes has increased from 108 million (1980) to 422 million (2014) worldwide, causing 1.6 million deaths in $2015[1,2]$. Diabetic patients are more susceptible to infection compared to nondiabetic counterparts, urinary tract infection being the most common bacterial infection encountered in these patients. Susceptibility of the infection in these patients increases with longer duration and greater severity of diabetes. This results in longer hospitalization, delay in recovery, and creates a sustainable burden in medical costs [3].

UTI is considered as the most common infectious disease affecting the socio-economic life of individual and society [4]. Despite the fact that both male and female are susceptible to UTI, women are more prone to UTI compared to male. Fifty percent of women will develop UTI in their life time and 1 in every 3 women requires antibiotics by the age of 24 due to UTI $[5,6]$. The main problem associated with UTI is its recurrence 
and persistence, which is mainly due to the presence of biofilm associated pathogen [7]. Biofilms are the microbial communities that are irreversibly associated with a surface and are enclosed in a self-produced extracellular polymeric matrix [8]. Bacteria within the biofilm behave differently from their planktonic counterparts especially in terms of antibiotics, which causes limitation in conventional antibiotic therapies [9]. It has been found that bacteria inside the biofilm are 10-1000 times more resistant to antimicrobial agents than the planktonic ones [10]. Increased incidence of diabetes worldwide in recent years and higher rates of antibiotic prescription, for UTI may further impose the development of antimicrobial-resistant urinary pathogens [3].

It has been noted that the etiological characteristics of uropathogen and their antibiotic resistance patterns may vary in different geographic locations, and with time $[4,11]$. In Nepal, limited published studies have been done to emphasize the antimicrobial resistance of uropathogen in diabetic patients and biofilm forming capacity of uropathogen. The main aim of this study is to determine the antimicrobial resistant uropathogen among diabetic and nondiabetic patients and detect the in-vitro biofilm forming capacity of uropathogen.

\section{Material and Methods}

2.1. Study Design and Population. This cross-sectional study was conducted from March 2015 to September 2015 at one of the Tertiary Hospitals, B\&B Hospital, located in Kathmandu valley, Nepal in collaboration with Department of Microbiology of Goldengate International College. A total of 1099 patients $($ diabetic $=182$ and nondiabetic $=917)$ suspected for UTI visiting the hospital during the study period were included in the study. Diabetic and nondiabetic patients were screened on the basis of blood glucose level. Those whose glucose profile cannot be traced or obtained were excluded from the study.

2.2. Ethical Consideration. The study was approved by Institute Review Committee (IRC) of the B\&B Hospital.

2.3. Sample Collection. Clean-catch midstream urine samples $(5-10 \mathrm{ml})$ were obtained from each patient in sterile screw-capped widemouthed container. The containers were well labeled with unique sample number, date, and time of collection. Samples were transferred to microbiology lab and processed within 1 hours of collection.

2.4. Sample Processing. The samples were processed by standard microbiological procedure [12]. Presence of $\geq 10^{5} \mathrm{CFU} / \mathrm{ml}$ of one and only type of organism was considered significant bacterial growth. Bacterial susceptibility to antimicrobial agents was determined by the modified KirbyBauer disc diffusion method on Muller Hilton agar according to CSLI guideline 2014 [13]. Antibiotics used were amikacin $(30 \mu \mathrm{g})$, amoxicillin $(10 \mu \mathrm{g})$, ciprofloxacin $(5 \mu \mathrm{g})$, ceftriaxone $(30 \mu \mathrm{g})$, cotrimoxazole $(1.25 / 23.75 \mu \mathrm{g})$, nitrofurantoin $(300 \mu \mathrm{g})$, piperacillin/tazobactum $(100 / 10 \mu \mathrm{g})$, imipenem $(10 \mu \mathrm{g})$, and tetracycline $(30 \mu \mathrm{g})$. Quality control strains of Escherichia coli (ATCC 25922) and Staphylococcus aureus (ATCC 25923) were used to validate the results of culture and antibiotic susceptibility test. Isolated organisms were classified as MDR and nonMDR on the basis of antimicrobial resistance pattern. Those isolates resistant to three or more than three groups of antimicrobial agents were classified as MDR [14].

In-vitro biofilm forming capacity of Escherichia coli was determined by Microtitre plate method as described by Christensen et al. [15]. The isolates were incubated in a 96-well Microtitre plate containing trypticase soya broth and glucose aerobically at $37^{\circ} \mathrm{C}$ for $18-24$ hours. Then the supernatant was discarded and washed with Phosphate Buffer Saline (PBS). The remaining attached bacteria were fixed with $300 \mu \mathrm{l}$ of ethanol. The OD values of the isolates that coat the wall of the wells were measured by using ELISA reader after staining with crystal violet. Biofilm producers were classified as negative (nonadherent), weak (weakly adherent), and high (strongly adherent) biofilm producers according to the observed OD values.

2.5. Statistical Analysis. Data were entered in Microsoft Office Excel and exported to IBMSPSS version 16.0 (SPSS Inc; Chicago, USA). The association between different variables and UTI was determined using chi-square test, frequency distribution, and univariate logistic regression analysis. An odds ratio with $95 \%$ confidence interval was considered statistically significant. $p$-value less than 0.05 was considered statistically significant.

\section{Results}

Total of 1099 urine samples were collected from diabetic patients (182) and nondiabetic patients (917). The mean age of diabetic patient suspecting UTI was $56.5 \pm 18.9$ and nondiabetic was $41.2 \pm 21.2$. The incidence of diabetes was higher in older age group i.e., patients over 65 years of age $(65,31.6 \%)$ compared to other groups (Figure 1).

Out of the total samples, 238 (21.7\%) showed significant bacterial growth. The incidence of UTI was found to be higher in female (odds ratio $[\mathrm{OR}]=1.518, p$-value $=0.006$, confidence interval $[\mathrm{CI}]=1.126-2.045)$ compared to male. This showed significant association of female with UTI. Similarly, the incidence of UTI was significantly higher in elderly patients $(\mathrm{OR}=2.283, p$-value $=0.005, \mathrm{CI}=1.286-4.055)$ and diabetic patients $(\mathrm{OR}=3.548, \quad p$-value $\leq 0.001, \quad \mathrm{CI}=2.527-4.983)$ compared to children and nondiabetic patients, respectively (Table 1).

Escherichia coli was the most common cause of urinary tract infection in both diabetic $(61,78.2 \%)$ and nondiabetic patients $(112,70.0 \%)$ (Table 2$)$. The prevalence of biofilm producing uropathogen was higher in both diabetic $(36,46.2 \%)$ and nondiabetic $(67,41.9 \%)$ groups (Figure 2$)$.

Of the 238 isolates, 103 (43.3\%) were found to be biofilm producers; 92 (38.7\%) were weak biofilm producers, and 11 (5.2\%) were strong biofilm producers (Table 3 ).

E. coli, the predominant cause of UTI showed higher percentage of resistance to amoxicillin followed by ciprofloxacin in both diabetic and nondiabetic patients. Least resistance rate 


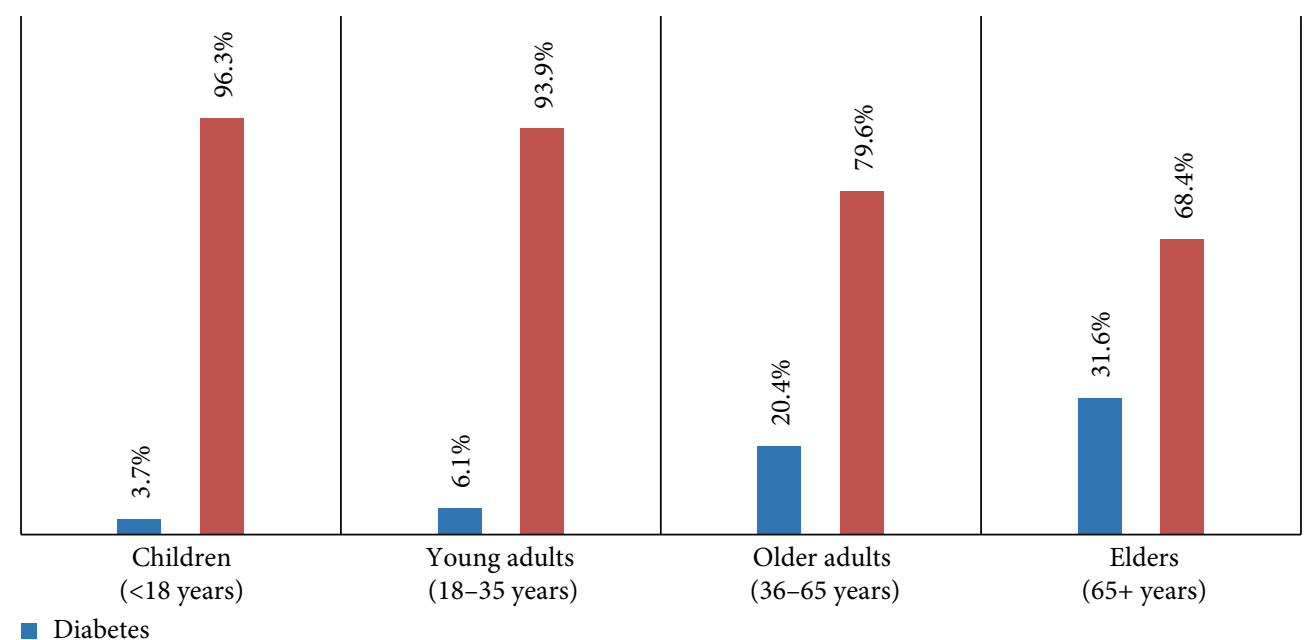

Diabetes

- Non-diabetes

FIGURE 1: Age distribution of diabetic and nondiabetic patients suspecting UTI.

TABLE 1: Association of UTI with other variables.

\begin{tabular}{|c|c|c|c|c|c|}
\hline & Growth & No growth & $p$-value & Odds ratio & $\begin{array}{c}95 \% \text { confidence } \\
\text { interval }\end{array}$ \\
\hline \multicolumn{6}{|l|}{ Sex } \\
\hline Male & $83(17.7 \%)$ & $386(82.3 \%)$ & & 1 & \\
\hline Female & $155(24.6 \%)$ & $475(75.4 \%)$ & 0.006 & 1.518 & $1.126-2.045$ \\
\hline \multicolumn{6}{|l|}{ Types of patient } \\
\hline Outpatient & $163(21.1 \%)$ & $610(78.9 \%)$ & & 1 & \\
\hline Inpatient & $75(23 \%)$ & $251(77 \%)$ & 0.481 & 1.118 & $0.820-1.525$ \\
\hline \multicolumn{6}{|l|}{ Age group } \\
\hline Children (<18 years) & $19(17.4 \%)$ & $90(82.6 \%)$ & & 1 & \\
\hline $\begin{array}{l}\text { Young adults } \\
\text { (18-35 years) }\end{array}$ & $62(18.8 \%)$ & $267(81.2 \%)$ & 0.742 & 1.100 & $0.624-1.939$ \\
\hline $\begin{array}{l}\text { Older adults } \\
\quad(36-65 \text { years })\end{array}$ & $90(19.8 \%)$ & $365(80.2 \%)$ & 0.577 & 1.168 & $0.677-2.016$ \\
\hline Elders (65+ years) & $67(32.5 \%)$ & $139(67.5 \%)$ & 0.005 & 2.283 & $1.286-4.055$ \\
\hline \multicolumn{6}{|l|}{ Disease } \\
\hline Non diabetic & $160(17.4 \%)$ & $757(82.6 \%)$ & & 1 & \\
\hline Diabetic & $78(42.9 \%)$ & $104(57.1 \%)$ & $<0.001$ & 3.548 & $2.527-4.983$ \\
\hline
\end{tabular}

TABLE 2: Distribution of uropathogens.

\begin{tabular}{lcc}
\hline Organism isolated & Diabetic $(n=78)$ & Nondiabetic $(n=160)$ \\
\hline Enterobacterales & & $70.0 \%$ \\
Escherichia coli & $78.2 \%$ & $4.4 \%$ \\
Klebsiella spp. & $9.0 \%$ & $2.5 \%$ \\
Enterobacter spp. & $1.3 \%$ & $0.6 \%$ \\
Citrobacter spp. & $0.0 \%$ & $3.1 \%$ \\
Proteus spp. & $1.3 \%$ & $0.6 \%$ \\
Morganella morganii & $0.0 \%$ & $5.0 \%$ \\
NonEnterobacterales & & $1.3 \%$ \\
Pseudomonas aeruginosa & $3.8 \%$ & $0.0 \%$ \\
Acinetobacter spp. & $0.0 \%$ & $7.5 \%$ \\
Gram positive & $0.0 \%$ & $5.0 \%$ \\
Non-Hemolytic streptococci & $5.1 \%$ & $1.3 \%$ \\
Coagulase negativeStaphylococcus & & \\
\hline
\end{tabular}


Non-diabetic

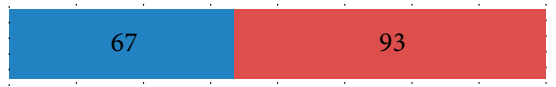

Diabetic

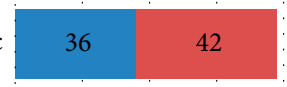

$\begin{array}{llllllllll}0 & 20 & 40 & 60 & 80 & 100 & 120 & 140 & 160 & 180\end{array}$

- Biofilm producer

- Biofilm non-producer

Figure 2: Biofilm production among diabetic and nondiabetic patients.

was observed with imipenem followed by nitrofurantoin, and amikacin. E. coli isolated from diabetic patients were found to be more resistant to most of the tested antibiotics compared to nondiabetics. However, no statistically significant relation was found between diabetic and antimicrobial resistance pattern among E. coli (Table 4).

No associations of the antibiotic sensitivity pattern were shown among other microorganisms because of the low isolation rate.

Biofilm producing E. coli showed comparatively high resistance rate to tested antimicrobial agents than nonbiofilm producing E. coli counterparts. The resistance rate of quinolones (CIP), third generation cephalosporin (CTR), and sulphonamide (COT) was statistically higher among biofilm producing E. coli (Table 5).

Though MDR E. coli was common in both biofilm producers and nonproducers, it was significantly higher in biofilm producer compared to nonbiofilm producing E. coli (Table 6).

\section{Discussion}

Diabetes is becoming epidemic and endemic problem in both developing and developed countries with higher prevalence and mortality in elderly population than in adults [16]. This study represented the higher incidence of diabetes in elderly population (31.6\%) compared to other age groups. The numbers of studies have evidenced the longer life expectancy in these days as the predisposing factor for diabetes. Decreases in insulin secretion, obesity, and vitamin $\mathrm{D}$ deficiency were known to contribute for developing diabetes in elderly population [16].

Out of 1099 urine samples requested for urine culture, $21.7 \%$ showed significant bacterial growth. This was in correspondence to the similarly conducted study in Kathmandu valley, Nepal $[17,18]$. Diabetic patients have higher odds of acquiring UTI compared to nondiabetic patients. Acharya et al. also showed a higher rate of UTI in diabetic patients $(34.5 \%)$, but at the lower rate compared to this study $[19,20]$. Niveditha et al., in their study, stated diabetes as the most common factor associated with UTI [21]. Nerve damage caused by high blood glucose level affects the ability of the bladder to sense the presence of urine in the bladder thus allowing the urine to stay in the bladder for longer time. The higher glucose level in the urine is known to improve the growth of bacteria in the urine and increased the probability of infection. In addition, the reduced blood circulation (due to prolonged diabetes mellitus) results in abnormalities of the host defense system, increasing the risk of developing infection [20].

Though UTI was found to be prevalent in both sexes and all age groups, it was higher in females $(\mathrm{OR}=1.518$, $p$-value $=0.006, \mathrm{CI}=1.126-2.045)$ compared to male. Different other studies also have shown higher rates of UTI in female compared to male $[22,23]$. Anatomical proximity of the urethra, sexual activity, and use of spermicidal contraceptives among female have been identified as the predisposing factor for UTI in female [24]. Similarly, the incidence of UTI was higher in elderly population $(\mathrm{OR}=2.283, p$-value $=0.005$, $\mathrm{CI}=1.286-4.055)$. Age-associated changes in immune system, exposure to nosocomial pathogens, increasing number of co-morbidity, urinary retention, high post void residual (PVR) urine, and prostatic hypertrophy in men have been postulated as risk factors in the elderly population [25]. On the other hand, increased unhygienic sexual activity in young groups increases the risk of UTI in adults.

E. coli was found to be the most common organism associated with UTI in both diabetic and nondiabetic. The ability of uropathogenic E. coli to cause infection is associated with expression of diverse virulence factors like fimbriae, siderophores, hemolysin, biofilm formation, etc. $[21,26]$. It has been reported that more than $65 \%$ of all bacterial infections are related to biofilm production; however, it also depends on the type of the infection and etiology [27]. Previous studies have also highlighted the role of biofilm formation in UTI $[28,29]$. Among all the bacterial isolates, $43.4 \%$ of the uropathogen were in-vitro positive for biofilm formation by the Microtitre plate assay method which corresponds to the study done by Abdagire et al. [30]. The incidence of biofilm production was found to be higher in both diabetic and nondiabetic patients with no statistical relation between them. Though biofilm forming capacity of uropathogen was found to be higher in in-vitro condition, it may differ in in-vivo condition. Hence, further study regarding the in-vivo biofilm forming capacity of uropathogen is necessary in case of relapses and treatment failure.

In this study E. coli showed highest percent of resistance to amoxicillin fallowed by ciprofloxacin and cotrimoxazole in both diabetic and nondiabetic patients. A similar study conducted at Tribhuvan University Teaching Hospital also showed amoxicillin as the least effective drug for gram negative bacteria [18]. Another study conducted at Kathmandu University School of Medical Science also presented ampicillin as the least effective drug for uropathogenic E. coli [19]. On the other side, imipenem, nitrofurantoin, and amikacin were found to be the most effective drug against uropathogenic E. coli. This implies the restricted use of amoxicillin and use of nitrofurantoin for uropathogenic E. coli, at least in the study area. Nitrofurantoin is known to be active against a wide range of uropathogen including multidrug resistant gram-negative bacilli and most of the $\beta$-lactamase-producing strains. However, in some medical conditions use of nitrofurantoin is restricted. Proteus spp., 
TABLE 3: Biofilm production of uropathogens.

\begin{tabular}{|c|c|c|c|}
\hline \multirow{2}{*}{ Organism isolated } & \multicolumn{3}{|c|}{ Biofilm } \\
\hline & Strongly adherent & Weakly adherent & Nonproducer \\
\hline \multicolumn{4}{|l|}{ Enterobacterales } \\
\hline Escherichia coli & 7 & 66 & 100 \\
\hline Klebsiella spp. & 3 & 6 & 5 \\
\hline Enterobacter spp. & 0 & 2 & 3 \\
\hline Citrobacter spp. & 0 & 0 & 1 \\
\hline Proteus spp. & 0 & 4 & 2 \\
\hline Morganella morgannei & 0 & 0 & 1 \\
\hline \multicolumn{4}{|l|}{ NonEnterobacterales } \\
\hline Pseudomonas aeruginosa & 1 & 5 & 5 \\
\hline Acinetobacter spp. & 0 & 1 & 1 \\
\hline \multicolumn{4}{|l|}{ Gram Positive } \\
\hline Non hemolytic streptococci & 0 & 6 & 10 \\
\hline Coagulase negative staphylococcus & 0 & 2 & 7 \\
\hline Total & 11 & 92 & 135 \\
\hline
\end{tabular}

TABLE 4: Antimicrobial resistance of E. coli isolated from diabetic and nondiabetic patients.

\begin{tabular}{|c|c|c|c|c|c|}
\hline \multirow{3}{*}{ Antimicrobial agents } & \multirow{2}{*}{\multicolumn{2}{|c|}{$\begin{array}{c}\text { Diabetic } \\
\text { E. coli }(n=61)\end{array}$}} & \multicolumn{2}{|c|}{ Nondiabetic } & \multirow{3}{*}{$P$-value } \\
\hline & & & & & \\
\hline & Resistance & $\%$ & Resistance & $\%$ & \\
\hline Amikacin & 9 & 14.8 & 11 & 9.8 & 0.332 \\
\hline Amoxicillin & 55 & 90.2 & 101 & 90.2 & 0.998 \\
\hline Ciprofloxacin & 42 & 68.9 & 65 & 58 & 0.162 \\
\hline Ceftriaxone & 33 & 54.1 & 64 & 57.1 & 0.7 \\
\hline Cotrimoxazole & 38 & 62.3 & 64 & 57.1 & 0.51 \\
\hline Nitrofurantoin & 7 & 11.5 & 14 & 12.5 & 0.884 \\
\hline Piperacillin-tazobactum & 23 & 37.7 & 35 & 31.3 & 0.39 \\
\hline Imipenem & 5 & 8.2 & 8 & 7.1 & 0.802 \\
\hline Tetracycline & 18 & 29.5 & 33 & 29.5 & 0.995 \\
\hline
\end{tabular}

TABLE 5: Antimicrobial resistance of biofilm producing and nonproducing E. coli.

\begin{tabular}{|c|c|c|c|c|c|}
\hline \multirow{2}{*}{ Antimicrobial agents } & \multicolumn{2}{|c|}{ Biofilm producer $E$. coli $(n=73)$} & \multicolumn{2}{|c|}{ Biofilm nonproducer $E$. coli $(n=100)$} & \multirow{2}{*}{$P$-value } \\
\hline & Frequency & $\%$ & Frequency & $\%$ & \\
\hline Amikacin & 9 & $12.3 \%$ & 11 & $11.0 \%$ & 0.787 \\
\hline Amoxicillin & 68 & $93.2 \%$ & 88 & $88.0 \%$ & 0.261 \\
\hline Ciprofloxacin & 55 & $75.3 \%$ & 52 & $52.0 \%$ & 0.002 \\
\hline Ceftriaxone & 51 & $69.9 \%$ & 46 & $46.0 \%$ & 0.002 \\
\hline Cotrimoxazole & 50 & $68.5 \%$ & 52 & $52.0 \%$ & 0.029 \\
\hline Nitrofurantoin & 9 & $12.3 \%$ & 12 & $12.0 \%$ & 0.0948 \\
\hline Piperacillin-tazobactum & 26 & $35.6 \%$ & 32 & $32.0 \%$ & 0.619 \\
\hline Imipenem & 7 & $9.6 \%$ & 6 & $6.0 \%$ & 0.376 \\
\hline Tetracycilin & 19 & $26.0 \%$ & 32 & $32.0 \%$ & 0.395 \\
\hline
\end{tabular}

Bold value indicates the significance difference between the antimicrobial resistance pattern of biofilm producing and non-producing $E$. coli for respective antimicrobial agents.

Seratia marcescens, and Pseudomonas aeruginosa are naturally resistant to nitrofurantoin. In these cases, other alternative drugs should be considered [31]. Since, nitrofurantoin and ciprofloxacin are commonly used for the empirical treatment of UTI in Nepal and this study showed high antimicrobial resistance to ciprofloxacin, nitrofurantoin is the only oral drug left. This calls for special attention and further studies regarding the antimicrobial resistance and antimicrobial use.

Antimicrobial resistance is an innate property of bacterial biofilm that may add complications to treatment [32]. The 
TABLE 6: MDR profiling of E. coli.

\begin{tabular}{|c|c|c|c|c|c|c|c|}
\hline & \multicolumn{2}{|c|}{ MDR } & \multicolumn{2}{|c|}{ NonMDR } & \multirow{2}{*}{$p$-value } & \multirow{2}{*}{ odds ratio } & \multirow{2}{*}{$\begin{array}{l}\text { 95\% confi- } \\
\text { dence interval }\end{array}$} \\
\hline & Frequency & $\%$ & Frequency & $\%$ & & & \\
\hline Biofilm producer $(n=73)$ & 58 & $79.5 \%$ & 15 & $20.5 \%$ & \multirow{2}{*}{0.015} & 2.37 & $1.181-4.757$ \\
\hline Biofilm nonproducer $(n=100)$ & 62 & $62.0 \%$ & 38 & $38.0 \%$ & & 1 & \\
\hline Diabetic $(n=61)$ & 45 & $73.8 \%$ & 16 & $26.2 \%$ & \multirow{2}{*}{0.354} & 1.387 & $1.694-2.775$ \\
\hline Nondiabetic $(n=112)$ & 75 & $67.0 \%$ & 37 & $33.0 \%$ & & 1 & \\
\hline
\end{tabular}

microbial biofilm responds poorly to conventional antibiotic therapy, thus resulting in persistence infections. Antibiotic resistance among biofilm producing $E$. coli was significantly higher for quinolones, trimethoprim-sulfamethoxazole, and third generation cephalosporin ceftriaxone. Increase in antimicrobial resistance within the biofilm arises from multiple factors including reduced bacterial growth rate, local alteration of the micro-environment that may impair activity of antimicrobial agents, EPS, and interbacterial interaction within the biofilm, which promote the spread of drug resistant markers and other virulence factors via gene transfer [8, 33]. Hence the screening of biofilm producing capacity of the isolate prior to antimicrobial treatment is crucial in effective management of the infection.

The isolates resistant to three or more than three classes of antibiotics were considered as MDR [14]. Niranjan and Malini have stated diabetes as the one of the major factors associated with the MDR E. coli [34]. Xavier et al. in their study have also reported high levels of MDR isolates in diabetic patients [35]. Nevertheless, our study showed a high degree of MDR E. coli in both diabetic (63.9\%) and nondiabetic groups $(60.7 \%)$. The high rates of antibiotic prescription in diabetic patients especially broad-spectrum antibiotics for UTI may be the cause for antibiotic resistant uropathogen [3]. This was in concurrence with the other studies done by Meiland et al., and Chakraborty et al. $[36,37]$. The difference in resistance pattern of the bacterial isolates may be due to difference in geographic region, the time of study conducted, and clinical practice. According to Sanchez et al., Alves et al., and Rao et al., biofilm formation increases the resistance profile of the organisms, and the strains that are capable of forming biofilm are mostly MDR phenotypes $[32,38,39]$. A similar report was observed in this study as MDR was about 2 times higher in biofilm forming $E$. coli than nonbiofilm forming $E$. coli $(p$-value $=0.015)$. Higher prevalence of MDR in biofilm producing strains may be due to transfer of resistant genes to the other organism that initially does not show such resistance [40]. Hence effective strategies for management of biofilm forming E. coli are crucial as it may lead to relapses in untreatable UTI.

\section{Conclusion}

Elderly population was found to be the most vulnerable group as diabetes and UTI are more common in this group. Furthermore, high antimicrobial resistant rate may impose a threat causing difficulty in treatment. Continuous surveillance of diabetes and UTI among elder population is necessary to ensure the rational use of antimicrobial agent for empirical and definitive treatment. Quinolones and trimethoprimsulfamethoxazole, commonly used in treatment of UTI was effective in only less than half number of E. coli. Along with this, the resistance rate for quinolone, trimethoprim-sulfamethoxazole and third generation cephalosporin ceftriaxone was significantly higher among biofilm producing isolates and most of the biofilm producing E. coli were found to be MDR. This calls for special attention as this may increase the antimicrobial resistance and chronicity of UTI. Further explorations genetically and by in-vivo study would improve our understanding to antimicrobial resistance and provide novel insights into the therapeutics and prevention against biofilm related infection.

\section{Data Availability}

The data used to support the findings of this study are available from the corresponding author upon request.

\section{Conflicts of Interest}

The authors declare that they have no conflicts of interests.

\section{Acknowledgments}

The authors would like to show our sincere gratitude to Goldengate International College, Battisputali, Kathmandu, Nepal, Mr. Binod Lekhak, Associate Professor, Tribhuvan University, and B\&B Hospital Pvt. Ltd., Gwarko, Lalitpur, Nepal. We would also like to thank all the patients, technical, and nontechnical staffs of B\&B Hospital Pvt. Ltd. for their help during the study.

\section{References}

[1] B. Gyawali, R. Sharma, D. Neupane, S. R. Mishra, E. van Teijlingen, and P. Kallestrup, "Prevalence of type 2 diabetes in Nepal: a systematic review and meta-analysis from 2000 to 2014," Global health action, vol. 8, no. 1, p. 29088, 2015.

[2] WHO, "Diabetes. 2019," World Health Organisation.

[3] W. Saliba, O. Nitzan, B. Chazan, and M. Elias, "Urinary tract infections in patients with type 2 diabetes mellitus: review of prevalence, diagnosis, and management," Diabetes, Metabolic Syndrome and Obesity: Targets and Therapy, vol. 8, p. 129, 2015.

[4] K. Md, A. Akter, F. Feroz, and A. Sunjukta, "Determination of antibiotic resistance pattern of biofilm producing pathogenic bacteria associated with UTI," International Journal of Drug Development \& Research, vol. 5, no. 4, pp. 312-319, 2013. 
[5] R. Vasudevan, "Urinary tract infection: an overview of the infection and the associated risk factors," Journal of Microbiology \& Experimentation, vol. 1, no. 2, p. 00008, 2014.

[6] A. Nerurkar, P. Solanky, and S. S. Naik, "Bacterial pathogens in urinary tract infection and antibiotic susceptibility pattern," Journal of Pharmaceutical and Biomedical Sciences, vol. 21, no. 21, 2012.

[7] K. Ejrnæs, M. Stegger, A. Reisner et al., "Characteristics of Escherichia coli causing persistence or relapse of urinary tract infections: phylogenetic groups, virulence factors and biofilm formation," Virulence, vol. 2, no. 6, pp. 528-537, 2011.

[8] P. Subramanian, "Antiobiotic resistance pattern of biofilm forming uropathogens isolated from catheterised patients in Pondicherry, India," Australasian Medical Journal, vol. 5, no. 7, pp. 344-348, 2012.

[9] U. Römling and C. Balsalobre, "Biofilm infections, their resilience to therapy and innovative treatment strategies," Journal of Internal Medicine, vol. 272, no. 6, pp. 541-561, 2012.

[10] H. Wu, C. Moser, H.-Z. Wang, N. Høiby, and Z.-J. Song, "Strategies for combating bacterial biofilm infections," International Journal of Oral Science, vol. 7, no. 1, pp. 1-7, 2015.

[11] A. Magyar, B. Köves, K. Nagy et al., "Spectrum and antibiotic resistance of uropathogens between 2004 and 2015 in a tertiary care hospital in Hungary," Journal of medical microbiology, vol. 66, no. 6, pp. 788-797, 2017.

[12] B. Forbes, D. Sahm, and A. Weissfeld, Study Guide for Bailey \& Scott's Diagnostic Microbiology, Mosby, USA, 12th edition, 2007.

[13] D. D. Rhoads, V. Sintchenko, C. A. Rauch, and L. Pantanowitz, "Clinical microbiology informatics," Clinical Microbiology Reviews, vol. 27, no. 4, pp. 1025-1047, 2014.

[14] A.-P. Magiorakos, A. Srinivasan, R. B. Carey et al., "Multidrugresistant, extensively drug-resistant and pandrug-resistant bacteria: an international expert proposal for interim standard definitions for acquired resistance," Clinical Microbiology and Infection, vol. 18, no. 3, pp. 268-281, 2012.

[15] G. D. Christensen, W. A. Simpson, J. J. Younger et al., "Adherence of coagulase-negative staphylococci to plastic tissue culture plates: a quantitative model for the adherence of staphylococci to medical devices," Journal of Microbiology, vol. 22, no. 6, pp. 996-1006, 1985.

[16] F. Chentli, S. Azzoug, and S. Mahgoun, "Diabetes mellitus in elderly," Indian Journal of Endocrinology and Metabolism, vol. 19, no. 6, p. 744, 2015.

[17] Y. P. Joshi, S. Shrestha, R. Kabir, A. Thapa, P. Upreti, and S. Shrestha, "Urinary tract infections and antibiotic susceptibility among the patients attending B\&D hospital of Lalitpur, Nepal," Asian Journal of Medical Sciences, vol. 7, no. 5, pp. 47-51, 2016.

[18] H. P. Kattel, A. Jyoti, K. M. Shyam, and R. Basista, "Bacteriology of urinary tract infection among patients attending tribhuvan university teaching hospital Kathmandu, Nepal," Journal of Nepal Association for Medical Laboratory Sciences, vol. 9, no. 1, pp. 25-29, 2008.

[19] D. Acharya, B. Bogati, G. Shrestha, and P. Gyawali, "Diabetes mellitus and urinary tract infection: spectrum of uropathogens and their antibiotic sensitivity," Journal of Manmohan Memorial Institute of Health Sciences, vol. 1, no. 4, pp. 24-28, 2015.

[20] M. Sewify, S. Nair, S. Warsame et al., "Prevalence of urinary tract infection and antimicrobial susceptibility among diabetic patients with controlled and uncontrolled glycemia in Kuwait," Journal of Diabetes Research, vol. 2016, pp. 1-7, 2016.
[21] S. N. Niveditha, "The isolation and the biofilm formation of uropathogens in the patients with catheter associated urinary tract infections (UTIs)," Journal of Clinical and Diagnostic Research, vol. 6, no. 9, pp. 1478-1482, 2012.

[22] K. Khadka, "Incidence of urinary tract infection among the patients visiting western regional hospital, Pokhara, Nepal," JHAS, vol. 2, no. 1, pp. 35-7, 2012.

[23] B. Pradhan and S. Pradhan, "Prevalence of urinary tract infection and antibiotic susceptibility pattern to urinary pathogens in kathmandu medical college and teaching hospital, Duwakot," Birat Journal of Health Sciences, vol. 2, no. 1, pp. 134-137, 2017.

[24] N. Tanko, "Retrospective studies on the prevalence of uropathogens in sokoto metropolis," African Journal of Microbiology Research, vol. 9, no. 20, pp. 1366-1370, 2015.

[25] T. A. Rowe and M. Juthani-Mehta, "Urinary tract infection in older adults," Aging Health, vol. 9, no. 5, pp. 519-528, 2013.

[26] J. Faghri, R. Dehbanipour, S. Rastaghi, M. Sedighi, and N. Maleki, "High prevalence of multidrug-resistance uropathogenic Escherichia coli strains, isfahan, Iran," Journal of Natural Science, Biology, and Medicine, vol. 7, no. 1, p. 22, 2016.

[27] L. Ghellai, H. Hassaine, N. Klouche et al., "Detection of biofilm formation of a collection of fifty strains of Staphylococcus aureus isolated in algeria at the university hospital of tlemcen," Journal of Bacteriology Research, vol. 6, no. 1, pp. 1-6, 2014.

[28] P. Tenke, B. Köves, K. Nagy et al., "Update on biofilm infections in the urinary tract," World Journal of Urology, vol. 30, no. 1, pp. 51-57, 2012.

[29] M. R. Hall, E. McGillicuddy, and L. J. Kaplan, "Biofilm: basic principles, pathophysiology, and implications for clinicians," Surgical Infections, vol. 15, no. 1, pp. 1-7, 2014.

[30] N. V. Abdagire, V. V. Chincholkar, D. M. Kulkarni, S. L. Nilekar, and S. V. Birajdar, "Biofilm production among uropathogenes and their antibiogram," International Journal of Pharma and Bio Sciences, vol. 5, no. 1, pp. 261-266, 2014.

[31] J. A. McKinnnel and L. G. Miller, "Nitrofurantoin: preferred empiric therapy for community-acquired lower urinary tract infections: in response," Mayo Clinic Proceedings, vol. 86, no. 12, p. 1244, 2011.

[32] C. J. Sanchez, K. Mende, M. L. Beckius et al., "Biofilm formation by clinical isolates and the implications in chronic infections," BMC Infectious Diseases, vol. 13, no. 1, p. 47, 2013.

[33] M. Kostakioti, M. Hadjifrangiskou, and S. J. Hultgren, "Bacterial biofilms: development, dispersal, and therapeutic strategies in the dawn of the postantibiotic era," Cold Spring Harbor Perspectives in Medicine, vol. 3, no. 4, pp. a010306-a010306, 2013.

[34] V. Niranjan and A. Malini, "Antimicrobial resistance pattern in Escherichia coli causing urinary tract infection among inpatients," The Indian Journal of Medical Research, vol. 139, no. 6, pp. 945-948, 2014.

[35] W. Xavier, T. S. Mangalanandan, K. V. Ajit, K. Harish, and C. Gopi, "Emergence of multi drug resistant bacteria in diabetic patients with lower limb wounds," The Indian Journal of Medical Research, vol. 140, no. 3, pp. 435-437, 2014.

[36] R. Meiland, S. E. Geerlings, A. J. De Neeling, and A. I. M. Hoepelman, "Diabetes mellitus in itself is not a risk factor for antibiotic resistance in Escherichia coli isolated from patients with bacteriuria," Diabetic Medicine, vol. 21, no. 9, pp. 1032-1034, 2004.

[37] A. Chakraborty, S. Shenoy, P. Adhikari, V. Saralaya, and S. Rao, "Is diabetes mellitus an important risk factor for the antibiotic 
resistance in extraintestinal pathogenic Escherichia coli?," Indian Journal of Pathology and Microbiology, vol. 60, no. 4, p. 546, 2017.

[38] M. J. Alves, J. C. M. Barreira, I. Carvalho et al., "Propensity for biofilm formation by clinical isolates from urinary tract infections: developing a multifactorial predictive model to improve the antibiotherapy," Journal of Medical Microbiology, vol. 63, no. Pt_3, pp. 471-477, 2014.

[39] K. Prashanth, R. S. Rao, R. U. Karthika et al., "Correlation between biofilm production and multiple drug resistance in imipenem resistant clinical isolates of Acinetobacter baumannii," Indian Journal of Medical Microbiology, vol. 26, no. 4, p. 333, 2008.

[40] P. Bhattacharyya, J. Gurung, A. B. Khyriem, A. Banik, W. V. Lyngdoh, and B. Choudhury, "Association of biofilm production with multidrug resistance among clinical isolates of Acinetobacter baumannii and Pseudomonas aeruginosa from intensive care unit," Indian Journal of Critical Care Medicine, vol. 17, no. 4, pp. 214-218, 2013. 


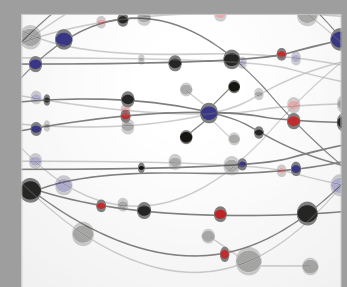

The Scientific World Journal
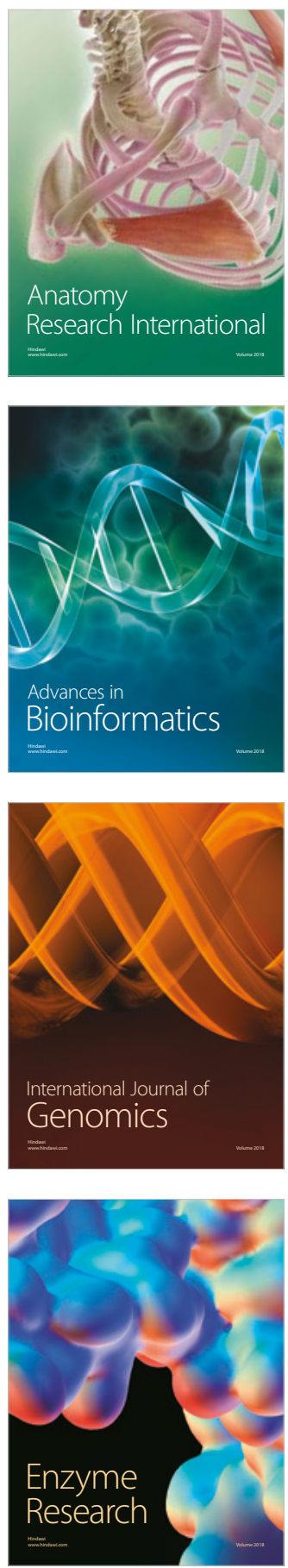
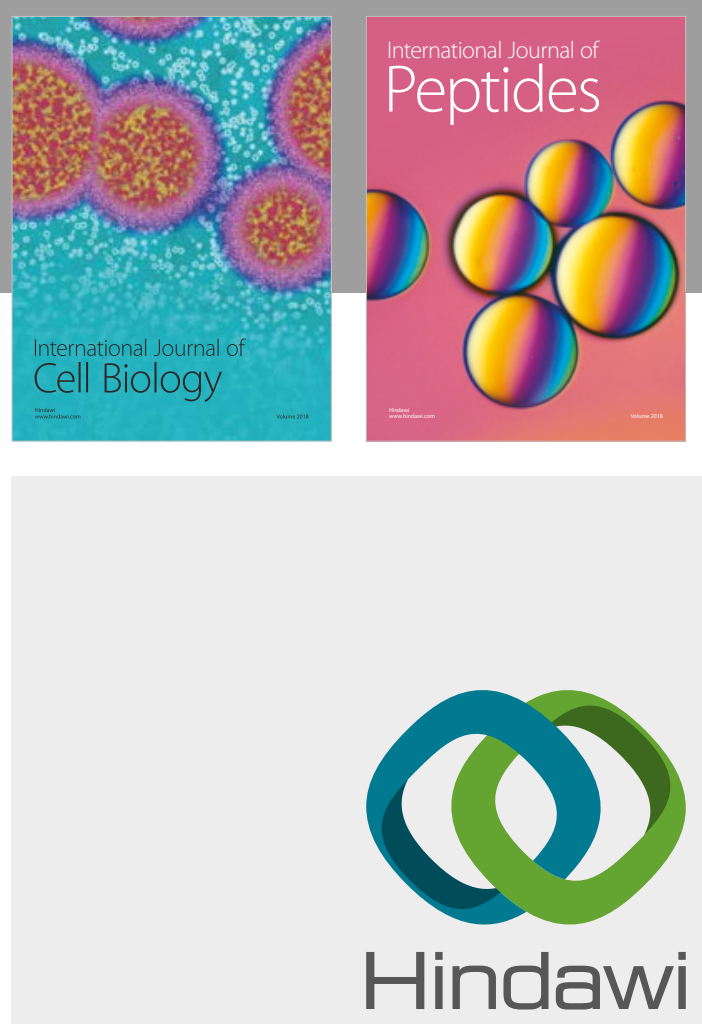

Submit your manuscripts at

www.hindawi.com
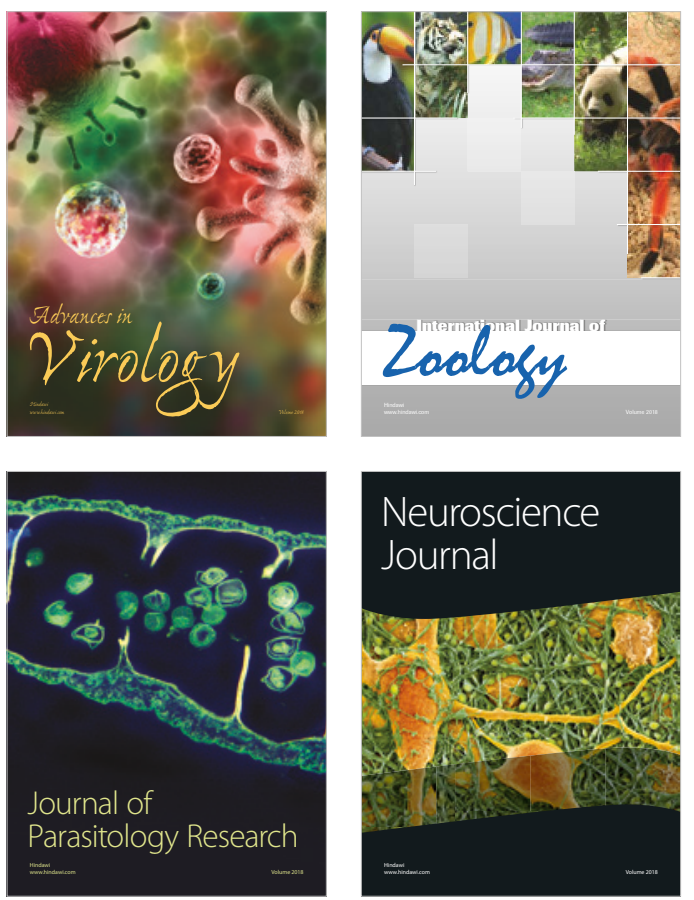
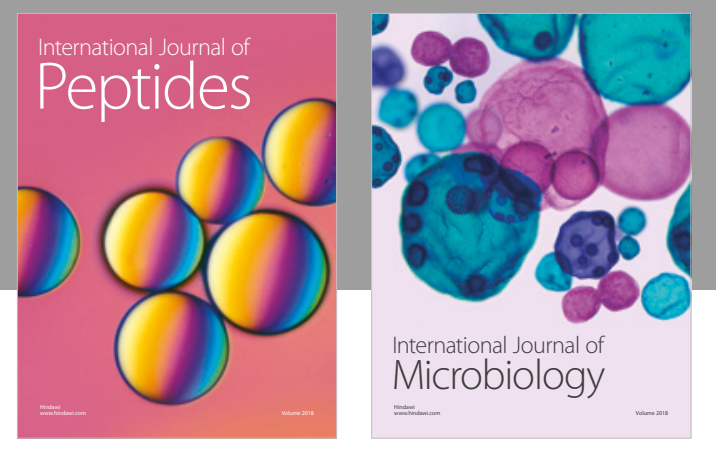

nternational Journal of Microbiology
Journal of
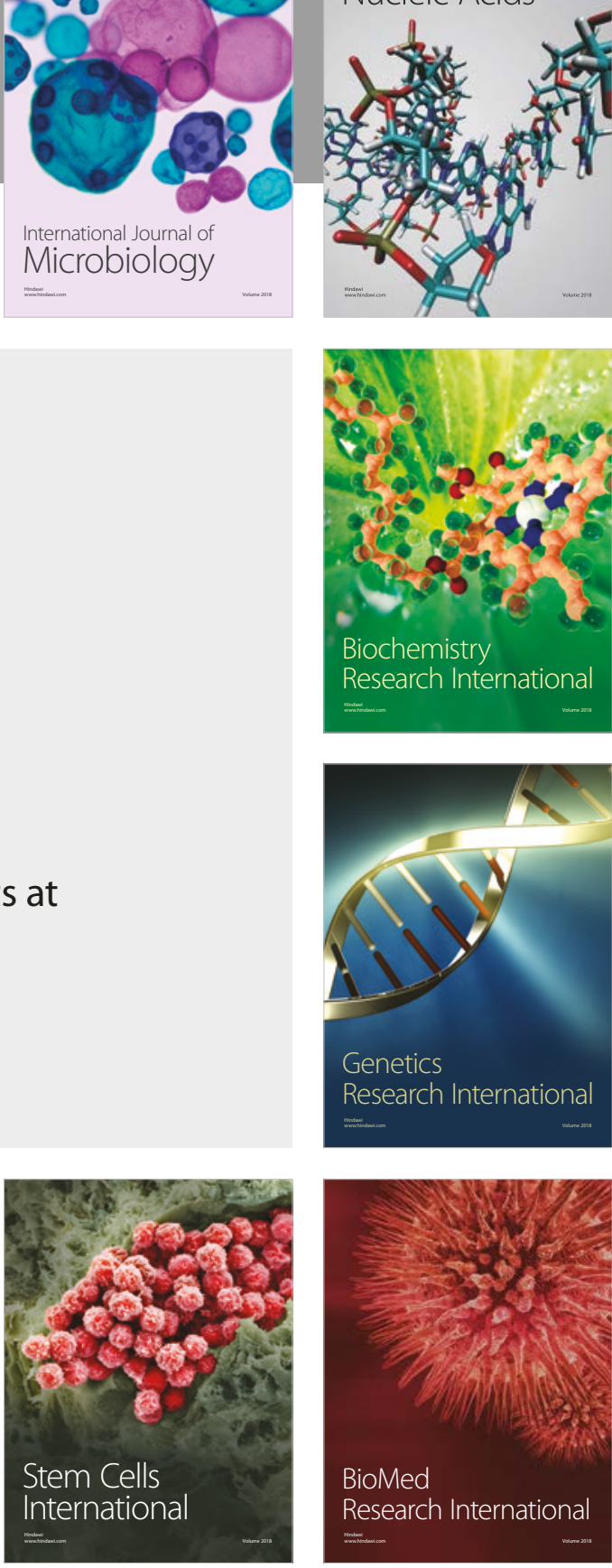
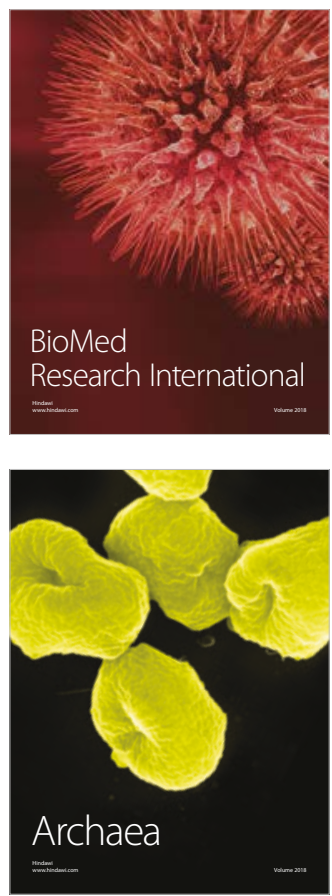